\title{
Evaluating the accuracy of DNA stable isotope probing
}

\author{
Nicholas D. Youngblut and Daniel H. Buckley* \\ School of Integrative Plant Science, Cornell University, 306 Tower Road, Ithaca, NY, 14853
}

*Corresponding author:

Daniel H. Buckley

Section of Soil and Crop Sciences

306 Tower Road

Ithaca, NY 14853

dbuckley@cornell.edu

(607) 255-1716

Running title: Evaluating the accuracy of DNA-SIP

Keywords: SIP, DNA, method, rRNA, microbial, bacterial, community, functional 


\section{Originality-Significance Statement}

2 By combining DNA Stable Isotope Probing (DNA-SIP) with multiplexed high throughput

3 DNA sequencing (HTS-DNA-SIP), it is now possible to identify patterns of isotope

4 incorporation for thousands of microbial taxa. HTS-DNA-SIP has enormous potential to

5 reveal patterns of carbon and nitrogen exchange within microbial food webs. A current

6 limitation is that, due to the expense of these experiments, it has been impossible to

7 evaluate the accuracy of DNA-SIP methods. We have developed a model that simulates

8 DNA-SIP data, and we use the model to systematically evaluate and validate the accuracy of

9 DNA-SIP analyses. This model can determine the analytical accuracy of DNA-SIP

10 experiments in a range of contexts. Furthermore, the ability to predict experimental

11 outcomes, as a function of experimental design and community characteristics, should be of

12 great use in the design and interpretation DNA-SIP experiments.

\section{Summary}

14 DNA Stable isotope probing (DNA-SIP) is a powerful method that identifies in situ isotope

15 assimilation by microbial taxa. Combining DNA-SIP with multiplexed high throughput DNA

16 sequencing (HTS-DNA-SIP) creates the potential to map in situ assimilation dynamics for

17 thousands of microbial taxonomic units. However, the accuracy of methods for analyzing

18 DNA-SIP data has never been evaluated. We have developed a toolset (SIPSim) for

19 simulating HTS-DNA-SIP datasets and evaluating the accuracy of methods for analyzing

20 HTS-DNA-SIP data. We evaluated two different approaches to analyzing HTS-DNA-SIP data:

21 "high resolution stable isotope probing" (HR-SIP) and "quantitative stable isotope probing"

22 (q-SIP). HR-SIP was highly specific and moderately sensitive, with very few false positives 
23 but potential for false negatives. In contrast, q-SIP had fewer false negatives but many false

24 positives. We also found HR-SIP more robust than q-SIP with respect to experimental

25 variance. Furthermore, we found that the detection sensitivity of HTS-DNA-SIP can be

26 increased without compromising specificity by evaluating evidence of isotope

27 incorporation over multiple windows of buoyant density (MW-HR-SIP). SIPSim provides a

28 platform for determining the accuracy of HTS-DNA-SIP methods across a range of

29 experimental parameters, which will be useful in the design, analysis, and validation of

30 DNA-SIP experiments.

\section{Introduction}

33 Stable isotope probing of nucleic acids (DNA-SIP and RNA-SIP) is a powerful culture-

34 independent method for linking microbial metabolic functioning to taxonomic identity

35 (Radajewski et al., 2003). In particular, DNA-SIP has been used extensively to identify

36 microbial assimilation of various ${ }^{13} \mathrm{C}$ - and ${ }^{15} \mathrm{~N}$-labeled substrates in a multitude of

37 environments (Uhlík et al., 2009). DNA-SIP identifies microbes that assimilate isotope into

38 their DNA ("incorporators") by exploiting the increased buoyant density (BD) of

39 isotopically labeled ("heavy") DNA relative to unlabeled ("light") DNA. For example, fully

$40{ }^{13} \mathrm{C}$ - and ${ }^{15} \mathrm{~N}$-labeled DNA will increase in BD by 0.036 and $0.016 \mathrm{~g} \mathrm{ml}^{-1}$, respectively (Birnie

41 and Rickwood, 1978).

42

43 Ideally, isopycnic centrifugation could be used to completely separate labeled and

44 unlabeled DNA fragments based solely on this difference in BD. However, several factors

45 besides $\mathrm{BD}$ can impact the position of DNA in isopycnic gradients. For example, $\mathrm{G}+\mathrm{C}$ 
content variation across a single genome can produce unlabeled DNA fragments that vary

47 in BD by up to $0.03 \mathrm{~g} \mathrm{ml}^{-1}$, while $\mathrm{G}+\mathrm{C}$ content variation between microbial genomes can

48 cause the average BD of unlabeled DNA fragments to vary by up to $0.05 \mathrm{~g} \mathrm{ml}^{-1}$ (Youngblut

49 and Buckley, 2014). In addition, DNA in SIP experiments will often be partially labeled as a

50 consequence of isotope dilution from unlabeled endogenous substrates. Therefore, it is

51 unlikely that nucleic acid SIP experiments will ever achieve complete separation of labeled

52 and unlabeled DNA.

54 In the absence of complete separation between labeled and unlabeled DNA, isotope 55 incorporators must be identified using some statistical procedure suitable for comparing 56 the BD distributions of DNA fragments from labeled and unlabeled samples (Pepe-Ranney 57 et al., 2016a). The use of multiplexed high throughput sequencing with DNA-SIP ("HTS58 DNA-SIP") makes it possible to sequence SSU rRNA amplicons across many density 59 gradient fractions and simultaneously determine the BD distributions for thousands of 60 taxa. The problem then becomes one of identifying those taxa that have increased in BD in

61 the isotopically labeled samples relative to the corresponding unlabeled controls.

63 Different analytical approaches have been applied to HTS-DNA-SIP datasets to identify 64 changes in DNA BD in response to isotopic labeling. These include "high resolution stable 65 isotope probing" (HR-SIP) and "quantitative stable isotope probing" (q-SIP), which both 66 analyze SSU rRNA amplicons across numerous gradient fractions (Hungate et al., 2015;

67 Pepe-Ranney et al., 2016a; Pepe-Ranney et al., 2016b). However, these methods differ in 68 the statistical procedures used to detect taxa that incorporate isotopic label. HR-SIP 
69 identifies isotopically labeled taxa by evaluating the sequence composition of high density

70 "heavy" fractions using a differential abundance quantification framework that evaluates

71 sequence count data in isotopically labeled samples relative to their corresponding

72 unlabeled controls. Differential abundance between the "heavy" fractions of labeled and

73 control gradients is measured with DESeq2 (Love et al., 2014), which uses sophisticated

74 statistical methods to reduce technical error and increase analytical power for analysis of

75 microbiome data (McMurdie and Holmes, 2014). In a very different approach, q-SIP

76 transforms SSU rRNA relative abundance values by using qPCR estimates of total SSU rRNA

77 gene copies present within gradient fractions. These normalized data are used to estimate

78 average BD for each taxon across density gradients for both isotopically labeled samples

79 and corresponding unlabeled controls (Hungate et al., 2015). Incorporators are then

80 determined by using a permutation procedure to identify those taxa whose BD shifts are

81 unlikely to occur as a result of chance.

83 While DNA-SIP is a powerful method for the discovery and characterization of

84 microorganisms in situ, systematic assessment of the specificity or sensitivity of this

85 method has not been performed. Empirical validations of DNA-SIP methods typically

86 include only one or a few organisms (Lueders et al., 2004; Buckley et al., 2007; Cupples et

87 al., 2007; Wawrik et al., 2009; Andeer et al., 2012), and such approaches do not adequately

88 replicate the complexity of the DNA fragment BD distributions expected in a typical DNA-

89 SIP experiment (Youngblut and Buckley, 2014). DNA-SIP experiments vary in the diversity

90 of the target community, DNA G + C content distribution, the number of incorporators,

91 incorporator relative abundance, and the atom \% excess of labeled DNA. Systematic 
92 evaluation of method accuracy should address the effects that all of these variables have on

93 the sensitivity and specificity of detecting isotope incorporators. Since DNA-SIP

94 experiments are costly, technically difficult, and laborious, it is not practical to perform

95 empirical assessment across this full range of variables.

97 Fortunately, the physics of isopycnic centrifugation have been well characterized

98 mathematically, and the behavior of individual DNA fragments in CsCl gradients is highly

99 reproducible and predictable from first principles (Meselson et al., 1957; Fritsch, 1975;

100 Birnie and Rickwood, 1978). In addition, genome sequences are available for thousands of

101 diverse microorganisms, and these genomes can be used to generate DNA fragments

102 representative of community DNA (Youngblut and Buckley 2014). Hence, we can simulate

103 realistic HTS-DNA-SIP data for in silico microbial communities that differ in diversity

104 (richness, evenness, and composition), where the relative abundance, genome $\mathrm{G}+\mathrm{C}$

105 content, and atom \% excess isotope are defined for discrete DNA fragments from every

106 genome. We have developed a computational toolset for simulating HTS-DNA-SIP data

107 (SIPSim) and used this simulation framework to systematically and objectively evaluate

108 how changes in key SIP experimental parameters affect HTS-DNA-SIP accuracy.

110 Results

111 Model validation and parameter estimation

112 The SIPSim model starts with a set of user-designated genomes and user-designated

113 experimental parameters (e.g. number of gradient fractions, desired community

114 characteristics, desired isotopic labeling characteristics) as described (see Experimental 
115 Procedures and Supporting Information). Briefly, the genomes are fragmented as would

116 occur during DNA extraction, isotopic labeling is applied to some number of genomes as

117 specified by the user, the BD distributions are determined for each DNA fragment and

118 fragment collections are then binned into gradient fractions, fragments are sampled from

119 each fraction as would occur during amplification and DNA sequencing of SSU rRNA genes,

120 and then the relative abundance is calculated for each OTU (Figure 1). The model produces

121 results that are highly similar to those observed in empirical experiments, including the

122 ability to detect DNA fragments throughout the density gradient (Figure 2).

123

124 The development of the simulation model was guided by established centrifugal theory and

125 by comparison of simulated results to empirical data (as in Experimental Procedures in

126 Supporting Information). First, we performed a simple evaluation of model performance by

127 recreating results from a prior DNA-SIP experiment with Methanosarcina barkeri MS and

128 Methylobacterium extorquens AM1 (Lueders et al., 2004) (Figure S1). Simulated DNA

129 distributions (both in terms of total DNA and SSU rRNA gene amplicon copies) significantly

130 and strongly correlated with the empirical data for both taxa $(p<0.003$ for all

131 comparisons; see Table S1). In addition, the simulated SSU rRNA gene amplicon-fragment

132 BD distributions were shifted $0.007 \mathrm{~g} \mathrm{ml}^{-1}$ toward the middle of the BD gradient relative to

133 the shotgun-fragments ("total DNA"), a phenomenon also observed in the empirical data.

134 This central tendency for SSU rRNA amplicon-fragments reflects $G+C$ conservation of the

135 rrn operon, as previously described (Youngblut and Buckley, 2014). 
137 Next, we determined whether the simulation accurately modeled variation in BD within

138 complex mixtures of unlabeled DNA by comparing simulation results to empirical results

139 obtained with unlabeled DNA from soil. For this purpose we used empirical data from an

140 experiment (Youngblut et al., in prep.) in which DNA was extracted from soil microcosms at

$1411,3,6,14,30$, and 48 days following the addition of an unlabeled carbon source mixture.

142 These six DNA samples were equilibrated in $\mathrm{CsCl}$ gradients, fractionated by BD, and SSU

143 rRNA gene amplicons were sequenced for $\sim 24$ fractions from each gradient. Simulation

144 input included 1147 microbial genomes (see Experimental Procedures), hence the soil data

145 was resampled to 1147 OTUs in order to standardize the richness of the simulated and

146 empirical data. Ideally, we could map SSU rRNA sequences from soil to all bacterial

147 genomes available in public databases, but genome composition can vary dramatically

148 across taxa that have identical SSU rRNA gene sequences. Since the genome sequences of

149 taxa in the empirical HTS-DNA-SIP dataset cannot be confidently assigned to genomes in

150 existing databases, a direct mapping of taxa (and their genomes) between the empirical

151 and simulated datasets was not possible. We therefore employed metrics that capture

152 variation in DNA fragment BD distributions within density gradients, and which thereby

153 allow for gradient to gradient comparison of DNA BD distributions (see Supporting

154 Information).

155

156 The empirical BD distributions (Figure S2) show that temporal change in soil mesocosm

157 community composition caused dramatic shifts in the Shannon diversity of 'heavy

158 fractions' even in the absence of isotopic labeling (Figure S2B), with heavy fraction

159 diversity increasing at later time points. Moreover, taxonomic similarity within a gradient 
160 is auto-correlated across the BD gradient (Figure S2C). Lastly, variance in amplicon

161 fragment BD is positively correlated with OTU relative abundance in the community

162 (Figure S2D), with highly abundant OTUs found throughout the CsCl gradient. We found

163 that the simulation model was able to recapitulate these results across a wide range of

164 parameter space, and that the variance between simulated and empirical results was less

165 than that observed between replicate empirical samples (Figure S3). We used these

166 comparisons to determine model parameter values (Table S2), which provided the best fit

167 to the actual behavior of DNA fragments in $\mathrm{CsCl}$ gradients (as described in Supporting

168 Information).

170 The influence of isotope incorporation on HTS-DNA-SIP accuracy

171 We hypothesized that both the number of taxa that incorporate isotope and the atom \%

172 isotope incorporation per taxon would substantially affect the accuracy of HTS-DNA-SIP

173 methods. To test these predictions, we simulated HTS-DNA-SIP datasets for both ${ }^{13} \mathrm{C}$ -

174 labeled samples and unlabeled controls (3 replicates of each), while varying both the

175 number of incorporators $(1,5,10,25$, or $50 \%$ of taxa) and the atom $\%$ isotope

176 incorporation for each taxon $\left(0,15,25,50\right.$, or 100 atom $\%$ excess $\left.{ }^{13} \mathrm{C}\right)$. Taxa in the control

177 were always set to $0 \%$ isotope incorporation. Each simulation was replicated 10 times,

178 with differing taxa randomly designated as incorporators in each replicate. We evaluated 4

179 methods used to analyze HTS-DNA-SIP data: Heavy-SIP, q-SIP, HR-SIP, and MW-HR-SIP.

180 Heavy-SIP involved simply identifying as incorporators all taxa observed in "heavy"

181 gradient fractions of labeled gradients, which provided a baseline of accuracy for the more

182 complex HTS-DNA-SIP analyses. q-SIP and HR-SIP were performed as described in Hungate 
183 et al., (2015) and Pepe-Ranney et al., (2016a), respectively. MW-HR-SIP was performed

184 similarly to HR-SIP, but with multiple overlapping "heavy" buoyant density windows (see

185 Experimental Procedures).

187 As expected, both the number of incorporators and the amount of isotope incorporated

188 affected accuracy (Figure 3). However, the effect of these parameters on specificity and

189 sensitivity varied depending on the analytical method (Figure 3). Specificity is the

190 proportion of true negatives observed out of all true negatives expected, and so specificity

191 declines in direct relation to an increase in the number of false positives. For example, a

192 specificity of 0.8 would generate 200 false positives in a sample of 1000 unlabeled taxa.

193 Specificity, as measured across a wide range in parameters, was highest for MW-HR-SIP (1

$194 \pm 0$; ave. \pm s.d.) and HR-SIP ( $1 \pm 0)$, substantially lower for q-SIP (0.88 \pm 0.06$)$, and very low

195 for Heavy-SIP $(0.28 \pm 0.16)$ (Figure 3).

197 Sensitivity is the fraction of true positives observed out of all true positives expected. For

198 example, a sensitivity of 0.7 means that a method failed to detect $30 \%$ of the incorporators

199 present. Both q-SIP and Heavy-SIP had relatively high sensitivity (median values of 0.91

200 and 0.93 , respectively), and the sensitivity of these methods was largely insensitive to the

201 atom \% excess of DNA and the number of incorporators (Figure 3). In contrast, the

202 sensitivities of both HR-SIP and MW-HR-SIP were highly responsive to the atom \% excess

203 of DNA, and the number of incorporators (Figure 3). For these methods, sensitivity

204 declined in proportion to the atom \% excess ${ }^{13} \mathrm{C}$ label in DNA. 
206 Balanced accuracy is calculated as the mean of specificity and sensitivity. We observed a

207 tradeoff in balanced accuracy in relation to the atom $\%$ excess ${ }^{13} \mathrm{C}$ of DNA. MW-HR-SIP had

208 the highest accuracy when $\%$ atom excess ${ }^{13} \mathrm{C}$ exceeded $50 \%$, but q-SIP had higher

209 accuracy at lower levels of isotope incorporation (Figure 3). This tradeoff in balanced

210 accuracy resulted from a difference in the tolerance for false positives. For example, MW-

211 HR-SIP produced nearly zero false positives but as a result of its high specificity, it lost

212 sensitivity at lower levels of isotope incorporation. In contrast, q-SIP detected labeled taxa

213 across a wider range of isotope incorporation, but it did so at the cost of a large number of

214 false positives.

216 The influence of community variation on HTS-DNA-SIP accuracy

217 All HTS-DNA-SIP analyses rely upon comparisons made between isotopically enriched

218 experimental treatments and their corresponding unlabeled controls. In real SIP

219 experiments the composition of replicate post incubation communities are likely to vary

220 somewhat as a result of sample heterogeneity and incubation effects. However, the

221 simulations described above assume random sampling from identical pre-fractionation

222 (post-incubation) community structures. We hypothesized that an increase in variation in

223 community composition between treatment and control samples would decrease the

224 accuracy of HTS-DNA-SIP analyses. To test this hypothesis, we generated simulations in

225 which isotope incorporation was held constant $\left(100\right.$ atom $\%$ excess ${ }^{13} \mathrm{C} ; 10 \%$ of OTUs are

226 incorporators) but beta-diversity was varied among 3 replicate treatment and 3 replicate

227 control samples. We varied beta-diversity in two ways: i) using permutation to vary the

228 rank abundance of a fixed proportion of community members and ii) varying the 
229 proportion of taxa shared between communities. For each simulation scenario, we

230 calculated the mean Bray-Curtis distance among communities in order to provide a real-

231 world metric for gauging the potential accuracy of actual DNA-SIP experiments.

233 As hypothesized, increased beta-diversity among samples had a substantial impact on the

234 accuracy of HTS-DNA-SIP methods (Figure 4). Accuracy was impacted more by the number

235 of taxa shared between samples than by differences in taxon abundance (Figure S4). The

236 sensitivity of q-SIP declined as beta-diversity increased, falling to $0.64 \pm 0.04$ (ave. \pm s.d.)

237 when samples shared $80 \%$ of their OTUs (Figure S4). In contrast, the sensitivities of MW-

238 HR-SIP and Heavy-SIP were least affected by changes in beta-diversity and these methods

239 had the highest sensitivity overall $(0.81 \pm 0.04$ and $0.82 \pm 0.03$ at $80 \%$ shared OTUs,

240 respectively; ave. \pm s.d.). Increasing the beta-diversity of samples had little effect on the

241 specificity of q-SIP but diminished slightly the specificity of HR-SIP and MW-HR-SIP (Figure

242 4). Despite these declines, HR-SIP and MW-HR-SIP maintained specificity that was greater

243 than or equal q-SIP and Heavy-SIP throughout most parameter space (Figure 4).

245 Of the methods evaluated, MW-HR-SIP had the highest balanced accuracy across the widest

246 range of parameters tested (Figure 4). Regardless, the balanced accuracy for MW-HR-SIP

247 was negatively affected by an increase in beta-diversity, falling from $0.98 \pm 0.02$ to $0.86 \pm$

2480.02 (ave. \pm s.d.) when the Bray-Curtis dissimilarity between samples was increased

249 beyond 0.5 (Figure 4). These results highlight the overall negative impact that sample-to-

250 sample variation has on HTS-DNA-SIP accuracy, and the importance of minimizing 
251 experimental variation between unlabeled controls and labeled treatments in SIP

252 experiments.

254 Using HTS-DNA-SIP data to quantify atom \% excess

255 So far we have focused on the accuracy of HTS-DNA-SIP methods with respect to the

256 identification of taxa that incorporate isotope into their DNA. However, changes in DNA BD

257 can also be used to quantify the isotope enrichment of DNA from particular taxa. Two

258 approaches have been used to evaluate isotope enrichment from HTS-SIP data: q-SIP and

$259 \Delta \mathrm{BD}$, with the latter being a complementary analysis to HR-SIP (Pepe-Ranney et al., 2016a).

260 Both $\triangle \mathrm{BD}$ and q-SIP derive quantitative estimates from measuring taxon BD shifts (and

261 thus atom \% excess) in the labeled treatment gradient(s) versus their unlabeled

262 counterparts. The $\triangle \mathrm{BD}$ method attempts to measure the extent of the BD shift directly from

263 the compositional sequence data, while q-SIP utilizes relative abundances transformed by

264 qPCR counts of total SSU rRNA copies. Therefore, $\triangle B D$ accuracy likely suffers from

265 compositional effects inherent to HTS datasets, while q-SIP accuracy is dependent on qPCR

266 accuracy and variation.

268 We assessed the quantification accuracy of both methods using the simulations described

269 previously, where either the amount of isotope incorporation or sample beta-diversity was

270 varied. We found that $\triangle \mathrm{BD}$ produced estimates of isotope incorporation that were closer on

271 average to the true value compared to $\mathrm{q}$-SIP, but $\Delta \mathrm{BD}$ values had much higher variance

272 than q-SIP estimates (Figure 5). Furthermore, the variance in $\Delta \mathrm{BD}$ atom $\%$ excess ${ }^{13} \mathrm{C}$

273 estimates increased substantially with even moderate increases in beta-diversity between 
274 samples, while the q-SIP estimations were largely invariant across the simulation

275 parameter space (Figure S5A). However, mean q-SIP values consistently underestimated

276 the true ${ }^{13} \mathrm{C}$ atom $\%$ excess by $30.2-39.2 \%$ (Figure 5B). Overall, quantitative estimates of

277 isotope incorporation for individual taxa were less variable with q-SIP, though q-SIP

278 consistently miss-estimated actual levels of isotope enrichment.

280 Discussion

281 Our simulation framework (SIPSim) provides a tractable platform for evaluating the

282 accuracy of DNA-SIP methods and for developing new methods to analyze DNA-SIP data.

283 Given the laborious nature of DNA-SIP experiments, it is impossible to use empirical

284 analyses with mock communities to evaluate the range of parameter values that can be

285 investigated through simulation. In addition, both the physics of density gradient

286 centrifugation and the physical properties of genomic DNA are well established, making the

287 simulation of DNA-SIP data both tractable and reliable. Without rigorous assessment of

288 DNA-SIP methods, it is difficult to determine the likelihood of false negatives (Type II error)

289 and false positives (Type I error) across the wide range of experimental conditions in

290 which DNA-SIP has been employed in the literature. Issues of Type I and Type II statistical

291 error are compounded in the analysis of HTS-DNA-SIP by the nature of HTS data, where it

292 is necessary to make many thousands of comparisons to identify those OTUs that change in

293 response to treatment. This multiple comparison problem has major implications for

294 statistical power and the likelihood of false detection (Paulson et al., 2013). We have used

295 SIPSim to test the effects of multiple parameters on the accuracy of current methods for

296 analyzing HTS-DNA-SIP data. Furthermore we used observations from the model to 
297 develop MW-HR-SIP, an analytical approach with balanced accuracy higher than any other

298 current method, with a higher sensitivity than HR-SIP, a higher specificity than q-SIP and

299 Heavy-SIP, and a higher robustness to inter-sample beta-diversity than all other currently

300 available methods.

302 Although both HR-SIP and q-SIP use high throughput SSU rRNA amplicon sequencing of

303 many gradient fractions, their different approaches for detecting isotope incorporators

304 result in substantial differences in sensitivity and specificity. In q-SIP, taxon relative

305 abundance is transformed using qPCR data to estimate counts of SSU rRNA gene copies

306 across gradient fractions; however, this approach resulted in a large number of false

307 positives ( $8 \pm 0.3$ to $15 \pm 0.7 \%$ of the unlabeled taxa evaluated were misidentified as

308 labeled; Figure 3 and Figure 4). Moreover, the number of false positives detected by q-SIP

309 increased dramatically in response to variation in community structure between samples

310 (Figure 4). In contrast, HR-SIP had negligible false positives under a wide range of

311 parameters (Figure 3 and Figure 4), but had lower sensitivity (more false negatives) than

312 other methods. However, we found that the sensitivity of HR-SIP was improved without

313 compromising specificity by using a multi-window analysis (MW-HR-SIP) in place of the

314 single window analysis used in HR-SIP. MW-HR-SIP has high sensitivity and specificity

315 across a range of experimental parameters, provided that the atom $\%$ excess ${ }^{13} \mathrm{C}$ of $\mathrm{DNA}$ is

316 in the range of $50-100 \%$ (Figure 3). At lower levels of isotope incorporation, q-SIP has

317 better sensitivity, but this sensitivity comes at the cost of detecting a large number of false

318 positives (i.e. low specificity). This tradeoff between specificity and sensitivity can be

319 contextualized by considering a community that contains 1045 unlabeled taxa and 55 
320 labeled taxa. If these 55 taxa are labeled at $50 \%$ atom excess ${ }^{13} \mathrm{C}$, both methods do a good

321 job detecting truly labeled taxa (MW-HR-SIP: $51 \pm 2$; q-SIP: $50 \pm 2$ ), but q-SIP detects far

322 more false positives (MW-HR-SIP: $0 \pm 1$; q-SIP: $126 \pm 8$ ). If these 55 taxa are instead labeled

323 at $25 \%$ atom excess ${ }^{13} \mathrm{C}$ then MW-HR-SIP detects fewer labeled taxa than q-SIP (MW-HR-

324 SIP: $33 \pm 3$; q-SIP: $50 \pm 2$ ), but q-SIP still detects far more falsely labeled taxa (MW-HR-SIP:

$3251 \pm 0$; q-SIP: $122 \pm 8$ ). In these examples, about $71 \%$ of the taxa identified by q-SIP as

326 labeled are actually unlabeled.

328 When considering the relative importance of sensitivity versus specificity for DNA-SIP

329 experiments, the ability to detect taxa that incorporate isotope is only useful if those

330 identifications can be made with high confidence (i.e. with a low number of false positives).

331 Therefore, based on our results, MW-HR-SIP is the most robust method for identifying

332 isotope incorporators from HTS-DNA-SIP data. In addition to its high specificity and better

333 ability to handle variance between replicate samples, MW-HR-SIP has the added advantage

334 of not requiring qPCR to be performed on each gradient fraction. It should be noted,

335 however, that the primary objective for which MW-HR-SIP was designed is the accurate

336 detection of labeled taxa, regardless of level of isotopic enrichment, while a major goal of q-

337 SIP is to quantify the atom \% excess of individual taxa.

339 In regards to methods used to quantify the atom \% excess of individual taxa from HTS-

340 DNA-SIP data, we found that the utility of q-SIP or $\triangle \mathrm{BD}$ varied depending on the hypothesis

341 being evaluated. $\triangle \mathrm{BD}$ produced more accurate estimates of mean ${ }^{13} \mathrm{C}$ atom $\%$ excess than q-

342 SIP (Figure 5 and Figure S5), and so this approach may be suitable when seeking to make 
343 relative comparisons in the degree of labeling between large groups of taxa (as described in

344 Pepe-Ranney, et al., 2016). However, the high variability of this approach causes $\Delta \mathrm{BD}$ to be

345 unreliable in determining differences in atom $\%$ excess ${ }^{13} \mathrm{C}$ at the scale of individual OTUs.

346 Alternatively, q-SIP produced much more stable estimates of atom $\%$ excess ${ }^{13} \mathrm{C}$ among

347 individual taxa, but the method resulted in systematic underestimates of isotope 348 incorporation.

350 The SIPSim framework makes it possible to both evaluate hypothetical outcomes of DNA-

351 SIP experiments before they are performed and to evaluate the accuracy of HTS-DNA-SIP

352 data analysis methods. For brevity, we have only focused on a few key variables that could

353 affect the accuracy of HTS-DNA-SIP methods. However, SIPSim can also be used to assess

354 the accuracy of DNA-SIP methods across a range of possible real-world scenarios. For

355 instance, spatial or population-level heterogeneity could result in taxa that are not

356 homogeneously labeled (Lennon and Jones, 2011). Such systematic heterogeneity in

357 labeling would manifest as "split" (bimodal or multimodal) distributions of DNA fragments

358 in an isopycnic gradient. It would be challenging to evaluate such scenarios empirically, but

359 SIPSim can be readily used to evaluate a range of such scenarios. SIPSim can also be used to

360 evaluate the effect of sequencing depth on the statistical power needed to resolve isotope

361 incorporation in rare taxa. Such information should be useful in planning HTS-DNA-SIP

362 experiments, to ensure that the experiment has a reasonable chance of success before it is

363 performed. Finally, SIPSim provides a toolkit for developing and improving analytical

364 methods used in DNA-SIP experiments. For example, a hybrid method that combines 
365 aspects of MW-HR-SIP and q-SIP may be able to produce robust incorporator identification

366 while also providing accurate estimates of the atom \% excess of individual taxa.

\section{Conclusion}

369 With our newly developed simulation toolset, we determined that MW-HR-SIP has the

370 highest accuracy of currently available methods for identifying taxa that have incorporated

371 isotope in HTS-DNA-SIP experiments. The use of MW-HR-SIP resulted in a negligible

372 number of false positives and its ability to detect true positives varied in relation to the

373 isotopic enrichment of DNA. Generally, we found that the specificity of all HTS-DNA-SIP

374 methods declined with increased beta-diversity among replicate samples. Thus, given that

375 accuracy declined most rapidly between a mean Bray-Curtis distance of 0 and 0.2 for all

376 methods evaluated (Figure 4), we recommend that researchers strive for mean Bray-Curtis

377 distances of $<0.2$ among replicate samples used in SIP experiments (i.e. between

378 treatments and their corresponding controls).

380 Experimental Procedures

381 Theory underlying the simulation framework

382 DNA stable isotope probing employs isopycnic centrifugation to separate isotopically

383 enriched ("heavy") DNA molecules from unlabeled ("light") DNA based on their differences

384 in buoyant density (BD). Isopycnic centrifugation is distinguished from other

385 centrifugation methods in that centrifugation is carried out long enough to both generate a

386 density gradient (typically using $\mathrm{CsCl}$ for DNA-SIP) and have all macromolecules of interest

387 reach sedimentation equilibrium, which is the point at which sedimentation rates equal 
rates of diffusion (Hearst and Schmid, 1973; Birnie and Rickwood, 1978). Empirical studies have shown that the average BD $(\rho)$ of a mixture of DNA molecules is linearly related to the average $\mathrm{G}+\mathrm{C}$ content for that collection of molecules:

$$
\rho=0.098[G+C]+1.66
$$

394 where $[G+C]$ is the mole fraction of $G+C$ content (Schildkraut et al., 1962; Birnie and 395 Rickwood, 1978). In addition, empirical studies have also shown that homogeneous 396 mixtures of DNA molecules form a Gaussian distribution in an isopycnic gradient when at 397 sedimentation equilibrium (Meselson et al., 1957; Fritsch, 1975). Therefore, in order to 398 model the BD distribution of a heterogeneous set of genomic DNA fragments, a Gaussian 399 distribution must be estimated for each homogeneous subset of molecules rather than 400 using discrete BD values (as described in Supporting Information). Based on the work of 401 Meselson and colleagues (Meselson et al., 1957), Fritsch (1975) derived an equation 402 describing time to reach sedimentation equilibrium, which can be reworked to calculate 403 the standard deviation $(\sigma)$ of the Gaussian distribution:

$$
\sigma=\frac{L}{e^{(\gamma-1.26)}}
$$

$$
\gamma=\frac{t \omega^{4} r_{p}^{2} s}{\beta^{\circ}\left(p_{p}-p_{m}\right)}
$$


409 where $L$ is the effective length of the gradient $(\mathrm{cm}), t$ is time in seconds, $\omega$ is the angular

410 velocity (radians $\mathrm{sec}^{-1}$ ), $r_{p}$ is the distance of the particle from the axis of rotation $(\mathrm{cm}), s$ is

411 the sedimentation coefficient of the particle, $\beta^{\circ}$ is the coefficient specific to the density

412 gradient medium (e.g. $\mathrm{CsCl}$ ); $p_{p}$ and $p_{m}$ are the maximum and minimum distances between

413 the gradient and axis of rotation (cm) (Fritsch, 1975). By assuming that sedimentation

414 equilibrium has been reached for all macromolecules of interest, Clay and colleagues

415 derived a simplified equation for determining $\sigma$ from the calculations in (Schmid and

416 Hearst, 1972):

$$
\sigma=\sqrt{\frac{\rho R T}{\beta^{2} G M_{C} l}}
$$

420 where $\rho$ is the BD of the particle, $R$ is the universal gas constant, $T$ is the temperature in

421 Kelvins, $\beta$ is a proportionality constant for aqueous salts of specific densities, $G$ is a

422 buoyancy factor as described in (Clay et al., 2003), $M_{C}$ is the molecular weight per base pair

423 of DNA, and $l$ is the fragment length (bp). For most DNA-SIP experiments, the assumption

424 of sedimentation equilibrium for all DNA fragments is likely to be unrealistic for relatively

425 short DNA fragments (e.g. $<4 \mathrm{~kb}$ ), given that the time to equilibrium rises dramatically

426 with decreasing fragment length (Meselson et al., 1957; Birnie and Rickwood, 1978;

427 Youngblut and Buckley, 2014). However, the ultracentrifugation durations used in typical

428 DNA-SIP experiments should still generally produce small $\sigma$ values for short DNA

429 fragments according to Eq. 2 (Neufeld et al., 2007). Therefore, equation Eq. 3 provides a 
430 good approximation for modeling the BD distribution of DNA in density gradients 431 generated in typical DNA-SIP experiments.

433 The distribution of a heterogeneous mixture of DNA fragments in an isopycnic gradient can

434 thus be modeled by integrating the Gaussian distributions of each homogeneous subset of 435 DNA fragments, where the mean of each Gaussian is determined by Eq. 1 and the standard 436 deviation derived from Eq. 3. In this way, the BD distribution for a given genome in an 437 isopycnic gradient can be modeled by the following steps: simulate genome fragmentation 438 resulting from DNA extraction, bin gDNA fragments with respect to length and $\mathrm{G}+\mathrm{C}$ 439 content, model Gaussian distribution for each fragment bin, and then integrate these 440 distributions to describe the cumulative DNA distribution in the gradient.

442 We found that empirical DNA fragment distributions differed from the expectations of a 443 strictly Gaussian model (Figure S2), and we determined that these differences could be 444 reconciled on the basis of established principles of fluid mechanics (as described below and 445 in Supporting Information). Based on empirical measurements, we found that most taxa 446 with relative abundances $>0.1 \%$ are detected in all gradient fractions when unlabeled DNA 447 is subjected to $\mathrm{CsCl}$ gradient centrifugation and SSU rRNA amplicon sequencing is 448 performed across a wide range of density gradient fractions (Figure S2). This observation 449 is in general congruence with observations in the literature (Birnie and Rickwood, 1978;

450 Lueders et al., 2004; Leigh et al., 2007), but it does not match the expectation that DNA 451 fragment distributions are strictly Gaussian, since the Gaussian model predicts that DNA 452 fragments should be undetectable (i.e. probability density $<1 \mathrm{e}^{-7}$ ) at either end of the 
453 density gradient (Figure S6). We explain the difference between observed and expected

454 DNA distributions as a function of fluid mechanics during gradient reorientation.

455

456 During isopycnic centrifugation, the buoyant density gradient forms perpendicular to the

457 axis of rotation (Figure S7), and gradient reorientation during centrifuge deceleration is

458 dramatic, especially for vertical rotors (Flamm et al., 1966). While the distortion of the BD

459 gradient during reorientation has been shown to be minimal in the aggregate (Fisher et al.,

460 1964; Flamm et al., 1966), the inevitable presence of a diffusive boundary layer along the

461 tube wall is sufficient to entrain quantities of DNA, which are small but should be readily

462 detectable by high throughput sequencing methods. The flow field that occurs during

463 gradient reorientation entrains along the tube wall a volume with a dimension

464 proportional to flow velocity, fluid viscosity, and surface topography (Tritton, 1977; Cohen

465 and Dowling, 2012). Following gradient reorientation, DNA from the entrained volume will

466 combine with DNA from the reoriented volume, thereby introducing a small amount of

467 non-BD-equilibrium DNA into each gradient fraction (Figure S7). The ability of the diffusive

468 boundary to introduce non-BD-equilibrium DNA into gradient fractions can be modeled as

469 a function of rotor geometry (Figure S7). Assuming sedimentation equilibrium, BD ( $\rho)$ can

470 be directly related to the distance from axis of rotation (Birnie and Rickwood, 1978):

471

$$
x=\sqrt{\left(p-p_{m}\right) \frac{2 \beta^{\circ}}{\omega^{2}}+r_{c}^{2}}
$$


474 From this calculation, the location of DNA molecules in the centrifuge tube, both during

475 centrifugation and fractionation, can be ascertained by using simple trigonometry along

476 with knowledge of centrifuge tube dimensions and angle to the axis of rotation. A full

477 description of the calculations along with an example can be found at

478 https://github.com/nick-youngblut/SIPSim. The fraction of a taxon's DNA fragments that

479 are in the boundary layer $\left(D_{t i}\right)$ is modeled as:

480

481

$$
D_{t i}=A_{t i} \gamma+\alpha
$$

482

483 where $A_{t i}$ is the pre-fractionation community relative abundance of taxon $t$ in gradient $i, \gamma$

484 is a weight parameter determining the contribution of $A_{t i}$ to $A_{b}$, and $\alpha$ is the baseline

485 fraction DNA in $A_{b}$.

486

487 Assimilation of the commonly used isotopes ${ }^{13} \mathrm{C}$ and ${ }^{15} \mathrm{~N}$ into genomic DNA produces linear

488 shifts in $\mathrm{BD}$, with a maximum shift of 0.036 and $0.016 \mathrm{~g} \mathrm{ml}^{-1}$, respectively (Birnie and

489 Rickwood, 1978). Thus the shift in BD ( $\rho)$ can be modeled as:

490

491

$$
\rho_{13 C}=I_{i, \max } A_{i}+\rho_{12 C}
$$

493 where $I_{i, \max }$ is the maximum possible BD shift if $100 \%$ atom excess for isotope $i, A$ is the 494 atom $\%$ excess of isotope $i$, and $\rho_{12 C}$ is the buoyant density at $0 \%$ atom excess. 
497 Based on the theory described above, our SIP data simulation framework simulates the

498 distribution of gDNA fragments in isopycnic gradients at sedimentation equilibrium.

499 Furthermore, it generates the HTS-DNA-SIP datasets obtained from fractionating isopycnic

500 gradient(s) and performing high throughput sequencing on many of the gradient fractions.

501 Our framework also implements all of the HTS-DNA-SIP analysis methods assessed in this

502 study (Heavy-SIP, HR-SIP, MW-HR-SIP, q-SIP, and $\Delta$ BD) and evaluates their accuracy of

503 identifying incorporators or quantifying BD shifts. An overview of our simulation

504 framework is shown in Figure 1.

505

506 Our simulation framework is a modular collection of steps that can be grouped in workflow

507 stages that are further broken down into steps (Figure 1). The input is a set of reference

508 genomes in fasta format and a text file designating the experimental design, which includes

509 the number of gradients for labeled treatments and unlabeled controls.

511 Stage 1 involves generating a BD distribution of gDNA fragments for each genome. Step 1a

512 involves simulating the pool of gDNA fragments that is extracted from SIP incubation

513 samples and then loaded into the isopycnic gradients. If amplicon sequence data (e.g. SSU

514 rRNA) is to be generated, amplicons from only the fragments containing the PCR template

515 ("amplicon-fragments") are sequenced, while shotgun metagenomic sequencing can target

516 all gDNA fragments ("shotgun-fragments"). If $\geq 1$ PCR primer set is provided, amplicon-

517 fragments are generated from genomic regions fully encompassing genome locations that

518 produced amplicons by in silico PCR. Alternatively, shotgun-fragments are randomly 
519 generated from all possible genomic locations. The fragment size distribution is user-

520 defined (Table S2).

521

522 As described in Eq. 1 \& 3, the length and $G+C$ content of a DNA fragment can be used to

523 calculate a probability distribution of its location in the gradient, assuming sedimentation

524 equilibrium. Step $1 \mathrm{~b}$ uses the fragments simulated in Step 1a to generate a 2-dimensional

525 Gaussian kernel density estimation (KDE) for each taxon, which describes the joint

526 probability of obtaining fragments with a certain length and $\mathrm{G}+\mathrm{C}$ content from that taxon.

527 From this 2D-KDE, a large number of [length, $G+C$ ] vectors can be simulated efficiently for

528 more precise estimations of the fragment BD distributions. Fragment BD distributions are

529 calculated for each taxon in Step 1c by sampling [length, $G+C$ ] vectors from the 2D-KDE

530 and calculating Gaussian distribution from each, where the mean is based on Eq. 1 and the

531 standard deviation based on Eq. 3. The collection of Gaussian distributions for all fragments

532 for each taxon is integrated into a BD distribution for all fragments of a taxon with Monte

533 Carlo error estimation, which involves sampling BD values from the collection of Gaussian

534 distributions and estimating a probability density function (PDF) of the fragment BD

535 distribution as a one-dimensional Gaussian KDE. The result is a list of KDEs, with each

536 describing the probability of detecting the gDNA fragments of a taxon at any point along the

537 isopycnic gradient. These fragment BD distributions are modified in steps $1 \mathrm{~d}$ and $1 \mathrm{e}$ by

538 adding diffusive boundary layer (DBL) effects (see Theory) and isotope incorporation,

539 respectively. The "smearing" due to DBL effects is modeled as a uniform distribution

540 describing the increased fragment BD uncertainty, and this uncertainty is integrated into

541 the fragment BD distributions by Monte Carlo error estimation as in Step 3b. The BD shift 
542 due to isotope incorporation is modeled in a similar manner, except BD uncertainty is a

543 result of inter- and intra-population variation in the amount of isotope incorporated.

544 Variation of isotope incorporation is modeled as a hierarchical set of mixture models

545 (weighted sets of standard distributions; such as two Gaussians), where the parameters for

546 intra-population mixture models that describe the amount of isotope incorporated by each

547 individual are themselves defined by inter-population mixture models that describe how

548 isotope incorporation varies among taxa.

550 Stage 2 involves simulating the isopycnic gradients for a particular experimental design.

551 Step 2a involves simulating the BD range size of each fraction of each gradient. Sizes are

552 drawn from a user-defined distribution. Step $2 b$ involves simulating the relative abundance

553 distribution of taxa in the gDNA pools loaded into each gradient ("pre-fractionation

554 communities"). The abundance distribution of each pre-fractionation community is user-

555 defined and can vary among gradients. Furthermore, the amount of taxa shared or rank-

556 abundances permutated among communities (i.e. the beta-diversity) is user-defined.

558 Stage 3 involves generating a HTS-DNA-SIP dataset based on the fragment BD distributions

559 simulated in Stage 1 along with the isopycnic gradient data generated in Stage 2. In Step 3a,

560 an OTU (taxon) abundance table is generated by sampling from the fragment BD

561 distributions of each taxon generated in Stage 1, with sampling depth determined by pre-

562 fractionation community abundances simulated in Step 2b. The subsampled fragments are

563 then binned into gradient fractions simulated in Step 2a. The resulting OTU table lists the

564 number of gDNA fragments of each taxon in each gradient fraction in each gradient. If the 
565 simulated fragments are amplicons, then PCR amplification efficiency biases are simulated

566 in Step 3b based on the PCR kinetic model described in Suzuki and Giovannoni (1996). The

567 model assumes that efficiencies decrease as the product concentration increases due to an

568 increased propensity of single stranded products to re-anneal to their homologous

569 complements. Sequence data is simulated in Step 3c by subsampling from the table of

570 fragment counts (the DNA fragment pool), which produces a final table ("HTS-DNA-SIP

571 dataset") of taxon relative abundances in each gradient fraction in each gradient.

572

\section{SIP data simulation framework parameters}

574 Unless stated otherwise, we made the following assumptions for all simulations in this

575 study. Community abundance distributions were simulated as lognormal distributions with

576 a mean of 10 and a standard deviation of 2 . All taxa were shared among communities, and

577 no rank-abundances were permuted (unless otherwise stated as for when evaluating beta-

578 diversity effects). The total number of fragments in each gradient was $1 \mathrm{e}^{9}$. Gradient

579 fragment BD range sizes were sampled from a normal distribution, with a mean of 0.004

580 and a standard deviation of 0.0015. SSU rRNA amplicon-fragments were simulated using

581 the V4-targeting 16S rRNA primers: 515F and 927R (5'-GTGYCAGCMGCMGCGGTRA-3'; 5'-

582 CCGYC AATTYMTTTRAGTTT-3'), as used by Pepe-Ranney and colleagues (Pepe-Ranney, et

583 al., 2016a). The amplicon-fragment size distribution was a left-skewed normal distribution

584 with a mean of $\sim 12 \mathrm{~kb}$, which is similar to size distributions produced from common bead

585 beating cell lysis methods (Kauffmann et al., 2004; Roh et al., 2006; Thakuria et al., 2008). A

586 total of $1 \mathrm{e}^{4}$ amplicon-fragments were simulated per genome, which equated to $>100 \mathrm{X}$

587 coverage for the genomic region of interest. Monte Carlo error estimation was conducted 
588 with $1 \mathrm{e}^{5}$ sampling replicates. Ultracentrifugation conditions were set as in Pepe-Ranney 589 and colleagues (Pepe-Ranney et al., 2016a), with a Beckman TLA-110 rotor spun at 5.5e ${ }^{4}$

$590 \mathrm{rpm}$ for 66 hours at $20^{\circ} \mathrm{C}$ and an average density gradient $1.7 \mathrm{~g} \mathrm{ml}^{-1}$. Inter-population

591 variation in isotope incorporation was binary (either $0 \%$ or X \% atom excess), and intra-

592 population variation was set to zero. Two key parameters were estimated from empirical

593 HTS-DNA-SIP data: the bandwidth (smoothing factor) for kernel density estimation, and

594 the gamma parameter in Eq. 5. See Table S2 for a full listing of simulation parameters.

595

596 Implementing HTS-DNA-SIP analyses

597 The HR-SIP method was performed as described in (Pepe-Ranney et al., 2016a; Pepe-

598 Ranney et al., 2016b). Briefly, we used a "heavy" BD window of 1.71-1.75 $\mathrm{g} \mathrm{ml}^{-1}$, a sparsity

599 cutoff of 0.25 (i.e. OTUs must be present in $>25 \%$ of samples), a $\log _{2}$ fold change null

600 threshold of 0.25 , and a false discovery rate cutoff of $10 \%$. $\Delta \mathrm{BD}$ was determined as

601 described by Pepe-Ranney and colleagues (Pepe-Ranney et al., 2016a), with OTU

602 abundances linearly interpolated across 20 evenly spaced values across the gradient BD

603 range.

604

605 We hypothesized that HR-SIP sensitivity could be improved by altering the "heavy" BD 606 window (1.71-1.75 $\mathrm{g} \mathrm{ml}^{-1}$ ) in which sequence composition is compared between treatment 607 and control. We evaluated different approaches and found that the analysis of multiple 608 windows (hereby called "MW-HR-SIP") resulted in a significant improvement in sensitivity 609 relative to HR-SIP. MW-HR-SIP evaluated sequence composition within BD windows of: $6101.70-1.73,1.72-1.75,1.74-1.77 \mathrm{~g} \mathrm{ml}^{-1}$ (Figure S8) while adjusting for multiple comparisons. 
612 q-SIP was conducted as described in Hungate and colleagues (Hungate et al., 2015), with 90

$613 \%$ confidence intervals calculated from 1000 bootstrap replicates. The variance among

614 qPCR replicates was modeled based on the qPCR data provided in Table S2 of Hungate et

615 al., (2015). Specifically, we found the qPCR count variance $\left(\sigma^{2}\right)$ to increase as a function of

616 the mean $(\mu)$. The following polynomial regression was found to best describe this

617 relationship and was used for simulating all qPCR count values:

$$
\sigma^{2}=5889+\mu+0.714 \mu^{2}
$$

621 where $\mu$ was set as the total number of simulated DNA fragments in the gradient fraction

622 (designated in the OTU table from Step 4a).

\section{Datasets}

625 The genome dataset used to simulate genomic DNA fragments was obtained from Genbank

626 (Benson et al., 2008). From a list of all bacterial genomes designated as "complete", one

627 representative was chosen per species in order to reduce the bias toward highly 628 represented species. We found the dataset to contain a rather high proportion $(\sim 12 \%)$ of 629 low $\mathrm{G}+\mathrm{C}$ organisms $(<30 \% \mathrm{G}+\mathrm{C})$; most of which were obligate endosymbionts. We 630 randomly sampled a subset of these low $G+C$ genomes in order to reduce the proportion 631 of low $\mathrm{G}+\mathrm{C}$ organisms to just $1 \%$ of the genome dataset. The resulting dataset consisted of 6321147 bacterial genomes. 
634 In order to simulate empirical data from Lueders and colleagues (Lueders et al., 2004), the

635 genome sequences of Methanosarcina barkeri MS and Methylobacterium extorquens AM1

636 were downloaded from Genbank. Amplicon-fragments were simulated with the primers

637 Ar109f (5'-ACKGCTCAGTAACACGT-3'), Ar915r (5'-GTGCTCCCCCGCCAATTCCT-3'), Ba519f

638 (5'-CAGCMGCCGCGGTAANWC-3'), and Ba907r (5'-CCGTCAATTCMTTTRAGTT-3'). Atom \%

639 excess was assumed to be $100 \%$, and isopycnic centrifugation conditions were simulated

640 as specified in Lueders et al., (2004).

641

642 For model evaluation (see Supporting Information - Results), we downloaded the genomes

643 Clostridium ljungdahlii DSM 13528, Escherichia coli 1303, and Streptomyces pratensis ATCC

64433331 from Genbank.

645

646 The HTS-DNA-SIP dataset from Youngblut and colleagues consisted of SSU rRNA MiSeq

647 sequences (V4 region) of $\sim 24$ fractions per gradient from 6 gradients of unlabeled controls

648 (Youngblut et al., in prep.). These data were subsampled to obtain a total richness equal to

649 the 1147 OTUs in our reference genome dataset. The sequence data is available from the

650 NCBI under BioProject PRJNA382302.

652 Software implementation

653 The SIP simulation framework was mostly written in Python v2.7.11, with some

654 accompanying code written in C++ v4.9.2 and R v3.2.3 (R Core Team, 2016). MFEprimer

655 v2.0 was used to perform in silico PCR (Qu et al., 2009). The software, along with

656 documentation and examples, can be found at https://github.com/nick-youngblut/SIPSim. 
657 All genomes were downloaded from Genbank with the R package genomes v2.12.0

658 (Stubben, 2014), and all data analysis was conducted in $\mathrm{R}$ with the following packages:

659 ggplot2 v2.1.0, dplyr v0.4.3, tidyr v 0.4.1, and cowplot v0.6.2.

660

661 Further methodological details are provided in the Supporting Information.

662

\section{Acknowledgements}

664 We thank Samuel Barnett for consultation on modeling the diffusive boundary layer effects

665 and Chuck Pepe-Ranney for helpful discussions on the modeling approach used in this

666 work. This material is based upon work supported by the Department of Energy, Office of

667 Biological \& Environmental Research Genomic Science Program under Award Numbers DE-

668 SC0010558 and DE-SC0004486.

\section{$670 \quad$ References}

671 Andeer, P., Strand, S.E., and Stahl, D.A. (2012) High-sensitivity stable-isotope probing by a

672 quantitative terminal restriction fragment length polymorphism protocol. Appl. Environ.

673 Microbiol. 78: 163-169.

674

675 Benson, D.A., Karsch-Mizrachi, I., Lipman, D.J., Ostell, J., and Wheeler, D.L. (2008) GenBank.

676 Nucleic Acids Res. 36: D25-D30.

678 Birnie, G.D. and Rickwood, D. (1978) Centrifugal separations in molecular and cell biology

679 Butterworths, Boston, MA. 
681 Buckley, D.H., Huangyutitham, V., Hsu, S.-F., and Nelson, T.A. (2007) Stable isotope probing 682 with ${ }^{15} \mathrm{~N}$ achieved by disentangling the effects of genome $\mathrm{G}+\mathrm{C}$ content and isotope 683 enrichment on DNA density. Appl. Environ. Microbiol. 73: 3189-3195.

684

685 Clay, O., Douady, C.J., Carels, N., Hughes, S., Bucciarelli, G., and Bernardi, G. (2003) Using 686 analytical ultracentrifugation to study compositional variation in vertebrate genomes. Eur.

687 Biophys. J. 32: 418-426.

688

689 Cohen, I.M. and Dowling, D.R. eds. (2012) Boundary layers and related topics. In Fluid 690 Mechanics (Fifth Edition). Kundu, Pijush K. (ed). Boston, USA: Academic Press, pp. 361691419.

692

693 Cupples, A.M., Shaffer, E.A., Chee-Sanford, J.C., and Sims, G.K. (2007) DNA buoyant density 694 shifts during ${ }^{15} \mathrm{~N}-\mathrm{DNA}$ stable isotope probing. Microbiol. Res. 162: 328-334.

695

696 Fisher, W.D., Cline, G.B., and Anderson, N.G. (1964) Density gradient centrifugation in angle697 head rotors. Anal. Biochem. 9: 477-482.

698

699 Flamm, W.G., Bond, H.E., and Burr, H.E. (1966) Density-Gradient centrifugation of DNA in a 700 fixed-angle rotor. Biochimica et Biophysica Acta (BBA) - Nucleic Acids and Protein Synthesis

701 129: $310-317$. 
703 Fritsch, A. (1975) Preparative density gradient centrifugations. Beckman Instrument

704 International, S.A. Geneva.

705

706 Hearst, J.E. and Schmid, C.W. (1973) Density gradient sedimentation equilibrium. In

707 Methods in Enzymology. Hirs, S.N.T. (ed). Boston, USA: Academic Press, pp. 111-127.

708

709 Hungate, B.A., Mau, R.L., Schwartz, E., Caporaso, J.G., Dijkstra, P., van Gestel, N., et al. (2015)

710 Quantitative microbial ecology through stable isotope probing. Appl. Environ. Microbiol. 81:

$711 \quad 7570-7581$.

712

713 Kauffmann, I.M., Schmitt, J., and Schmid, R.D. (2004) DNA isolation from soil samples for

714 cloning in different hosts. Appl. Microbiol. Biotechnol. 64: 665-670.

715

716 Leigh, M.B., Pellizari, V.H., Uhlík, O., Sutka, R., Rodrigues, J., Ostrom, N.E., et al. (2007)

717 Biphenyl-utilizing bacteria and their functional genes in a pine root zone contaminated

718 with polychlorinated biphenyls (PCBs). ISME J. 1: 134-148.

719

720 Lennon, J.T. and Jones, S.E. (2011) Microbial seed banks: the ecological and evolutionary

721 implications of dormancy. Nat. Rev. Microbiol. 9: 119-130.

722

723 Love, M.I., Huber, W., and Anders, S. (2014) Moderated estimation of fold change and

724 dispersion for RNA-seq data with DESeq2. Genome Biol. 15: 550.

725 
726 Lueders, T., Manefield, M., and Friedrich, M.W. (2004) Enhanced sensitivity of DNA- and

727 rRNA-based stable isotope probing by fractionation and quantitative analysis of isopycnic

728 centrifugation gradients. Environ. Microbiol. 6: 73-78.

729

730 mcmurdie, p.j. and holmes, s. (2014) Waste not, want not: why rarefying microbiome data

731 is inadmissible. PLoS Comput. Biol. 10.:

732

733 Meselson, M., Stahl, F.W., and Vinograd, J. (1957) Equilibrium sedimentation of

734 macromolecules in density gradients. Proceedings of the National Academy of Sciences 43:

$735 \quad 581-588$.

736

737 Neufeld, J.D., Vohra, J., Dumont, M.G., Lueders, T., Manefield, M., Friedrich, M.W., and

738 Murrell, J.C. (2007) DNA stable-isotope probing. Nat. Protoc. 2: 860-866.

739

740 Paulson, J.N., Stine, O.C., Bravo, H.C., and Pop, M. (2013) Differential abundance analysis for

741 microbial marker-gene surveys. Nat. Methods 10: 1200-1202.

742

743 Pepe-Ranney, C., Campbell, A.N., Koechli, C.N., Berthrong, S., and Buckley, D.H. (2016)

744 Unearthing the ecology of soil microorganisms using a high resolution DNA-SIP approach

745 to explore cellulose and xylose metabolism in soil. Front. Microbiol. 7: 703.

746

747 Pepe-Ranney, C., Koechli, C., Potrafka, R., Andam, C., Eggleston, E., Garcia-Pichel, F., and

748 Buckley, D.H. (2016) Non-cyanobacterial diazotrophs mediate dinitrogen fixation in 
749 biological soil crusts during early crust formation. ISME J. 10: 287-298.

750

751 Qu, W., Shen, Z., Zhao, D., Yang, Y., and Zhang, C. (2009) MFEprimer: multiple factor

752 evaluation of the specificity of PCR primers. Bioinformatics 25: 276-278.

753

754 Radajewski, S., McDonald, I.R., and Murrell, J.C. (2003) Stable-isotope probing of nucleic

755 acids: a window to the function of uncultured microorganisms. Curr. Opin. Biotechnol. 14:

$756 \quad 296-302$.

757

758 R Core Team (2016) R: A Language and Environment for Statistical Computing R

759 Foundation for Statistical Computing, Vienna, Austria.

760

761 Roh, C., Villatte, F., Kim, B.-G., and Schmid, R.D. (2006) Comparative study of methods for

762 extraction and purification of environmental DNA from soil and sludge samples. Appl.

763 Biochem. Biotechnol. 134: 97-112.

764

765 Schildkraut, C.L., Marmur, J., and Doty, P. (1962) Determination of the base composition of

766 deoxyribonucleic acid from its buoyant density in CsCl. J. Mol. Biol. 4: 430-443.

767

768 Schmid, C.W. and Hearst, J.E. (1972) Sedimentation equilibrium of DNA samples

769 heterogeneous in density. Biopolymers 11: 1913-1918.

770

771 Stubben, C. (2014) genomes: Genome sequencing project metadata. R Package version 
774 Suzuki, M.T., Giovannoni, S.J. (1996) Bias caused by template annealing in the amplification

775 of mixtures of 16S rRNA genes by PCR. Appl. Environ. Microbiol. 62: 625-630.

777 Thakuria, D., Schmidt, O., Mac Siúrtáin, M., Egan, D., and Doohan, F.M. (2008) Importance of

778 DNA quality in comparative soil microbial community structure analyses. Soil Biol.

779 Biochem. 40: 1390-1403.

780

781 Tritton, D.J. (1977) Boundary Layers and Related Topics. In Physical Fluid Dynamics.

782 Netherlands: Springer, pp. 101-118.

783

784 Uhlík, O., Jecná, K., Leigh, M.B., Macková, M., and Macek, T. (2009) DNA-based stable isotope

785 probing: a link between community structure and function. Sci. Total Environ. 407: 36117863619.

788 Wawrik, B., Callaghan, A.V., and Bronk, D.A. (2009) Use of inorganic and organic nitrogen

789 by Synechococcus spp. and diatoms on the west florida shelf as measured using stable

790 isotope probing. Appl. Environ. Microbiol. 75: 6662-6670.

791

792 Youngblut, N.D. and Buckley, D.H. (2014) Intra-genomic variation in G + C content and its

793 implications for DNA stable isotope probing. Environ. Microbiol. Rep. 6: 767-775. 


\section{$795 \quad$ Figure Legends}

796 Figure 1. The SIPSim simulation workflow involves three major stages, which are broken

797 down into multiple steps. Stage 1 involves generating a buoyant density distribution of

798 gDNA fragments for each genome. Stage 2 involves simulating the isopycnic gradients for a

799 particular experimental design. Stage 3 involves generating a HTS-DNA-SIP dataset based

800 on the fragment BD value distributions simulated in Stage 1 along with the isopycnic

801 gradient data generated in Stage 2. The output is a table ("HTS-DNA-SIP dataset") of taxon

802 relative abundances in each gradient fraction in each gradient. See Experimental Procedures

803 for a more detailed description of the simulation workflow.

804

805 Figure 2. Compositional effects can distort and obscure BD shifts resulting from ${ }^{13} \mathrm{C}$ isotope

806 incorporation. The plots show DNA fragment distributions resulting from simulation of

8071147 taxa (one color per taxon) within $\mathrm{CsCl}$ gradient pairs consisting of: a ${ }^{12} \mathrm{C}$-control

808 ("control") and a ${ }^{13} \mathrm{C}$-treatment ("treatment") gradient. For this simulation, all taxa in the

809 control gradient had $0 \%$ atom excess ${ }^{13} \mathrm{C}$, while $10 \%$ of taxa in the treatment gradient were

810 randomly assigned $100 \%$ atom excess ${ }^{13} \mathrm{C}$. "Pre-sequencing simulation" (top) and "Post-

811 sequencing simulation" (middle, and bottom) show fragment BD distributions before and

812 after simulating the effect of uniform random sampling which results from high throughput

813 sequencing of all gradient fractions at an equal number of sequences. The "absolute

814 abundance" (top and middle) indicates the number of DNA fragments from each taxon in

815 each gradient fraction, while "relative abundance" (bottom) indicates the relative

816 abundance of each taxon. Note that the top plot represents the actual amplicon-fragment

817 distributions in an isopycnic gradient at equilibrium, while the bottom plot represents the 
818 sampled fragment distributions obtained after high throughput sequencing. The dashed

819 vertical line is provided as a point of reference and designates the theoretical buoyant

820 density of an unlabeled DNA fragment with $50 \% \mathrm{G}+\mathrm{C}$ (as modeled in Eq. 1).

821

822 Figure 3. HTS-DNA-SIP methods vary in accuracy depending on the ${ }^{13} \mathrm{C}$ atom $\%$ excess of

823 DNA and the number of taxa that incorporate isotope. Points and bars represent means and

824 standard deviations, respectively ( $\mathrm{n}=10$ simulations). Specificity indicates the proportion

825 of true negatives that are identified correctly and it is used to quantify false positives.

826 Sensitivity indicates the proportion of labeled taxa (true positives) identified correctly.

827 Balanced accuracy is a function of both specificity and sensitivity. The $x$-axis indicates the

828 amount of ${ }^{13} \mathrm{C}$ isotope present in taxa that are labeled, and different colors are used to

829 indicate the percentage of taxa that have incorporated ${ }^{13} \mathrm{C}$ as indicated by the legend.

830

831 Figure 4. HTS-DNA-SIP methods differ in their sensitivity to community dissimilarity between

832 replicate samples. Beta diversity, expressed as Bray-Curtis dissimilarity, was varied

833 between simulated replicates (3 replicates each for ${ }^{12} \mathrm{C}$-control and ${ }^{13} \mathrm{C}$-treatment

834 gradients) to determine the effect that community dissimilarity between replicates has on

835 method accuracy. Variation in beta diversity was simulated by systematically varying two

836 parameters: the percent of taxa shared between replicate samples $(80,85,90,95$, or 100

$837 \%)$ and the percent of taxa whose rank abundances that were permuted $(0,5,10,15$, or 20

$838 \%$ ), with 10 simulation replicates for each parameter set. The blue lines are LOESS curves

839 fit to accuracy values for all simulations $(n=250)$, and the grey regions represent $99 \%$ 
840 confidence intervals. For all simulations, $10 \%$ of the community were incorporators $(100$

$841 \%$ atom excess ${ }^{13} \mathrm{C}$ ).

842

843 Figure 5. $\triangle B D$ and $q S I P$ vary in their accuracy at estimating ${ }^{13} C$ atom $\%$ excess of labeled DNA

844 fragments. (A) The accuracy of both methods declines as the amount of ${ }^{13} \mathrm{C}$ in DNA increases,

845 but accuracy is not affected by the percent of taxa that are labeled; values indicate the mean and

846 standard deviation ( $\mathrm{n}=10$ simulations). (B) Probability density plots indicate that estimates of

$847{ }^{13} \mathrm{C}$ atom $\%$ excess made using $\triangle B D$ have greater variance than those made using $q S I P$, but both

848 estimates systematically underestimate levels of isotope incorporation. Each vertical pair of

849 panels indicates the probability density for estimates made across different levels of isotope

850 incorporation $(15,25,50$, and 100 atom $\%$ excess $)$, and the dashed line indicates the actual level

851 of isotopic enrichment. For the calculation of probability density, $10 \%$ of taxa were labeled

852 using the level of enrichment indicated in each panel. 


\section{Basic model input}

Genome sequence dataset

fasta-formatted genome files

\section{Experimental design}

the number of gradients for labeled

and unlabeled treatments

\section{Simulating DNA fragment BD distributions}

1a) Genomic DNA fragments amplicon fragments (e.g., 16S rRNA) or shotgun metagenome fragments

\section{1b) DNA fragment length $\& \mathrm{G}+\mathrm{C}$} distributions

a 2-dimensional probability distribution of the key fragment properties

1c) DNA fragment BD probability distributions

fragment BD modeled as Gaussian

probability density functions

\section{1d) Diffusive boundary layer effects}

broadening of fragment $\mathrm{BD}$

distributions due to DBL "smearing"

\section{1e) Isotope incorporation}

shifting fragment $B D$ distributions due to inter- and intra-population isotope incorporation

\section{Simulating isopycnic gradients}

\begin{tabular}{|l|l|l|}
\hline $\begin{array}{l}\text { 2a) Gradient fractions } \\
\text { the BD range of each } \\
\text { fraction in each gradient }\end{array}$ & $\begin{array}{l}\text { 2b) Pre-fractionation communities } \\
\text { taxon abundances in community } \\
\text { DNA loaded into each gradient }\end{array}$ \\
\hline
\end{tabular}

\section{$\downarrow$}

Simulating HTS-DNA-SIP data

3a) Fragment count table

each taxon's amplicon- or shotgun-fragment count in each fraction

of each gradient

\section{3b) PCR products}

adjusted fragment counts based on PCR reaction efficiencies (only for amplicon-fragments)

\section{3c) Sequence data}

subsampling fragment counts in each gradient fraction

\section{$\downarrow$}

HTS-DNA-SIP dataset

OTU abundance table

(1 sample per gradient fraction) 


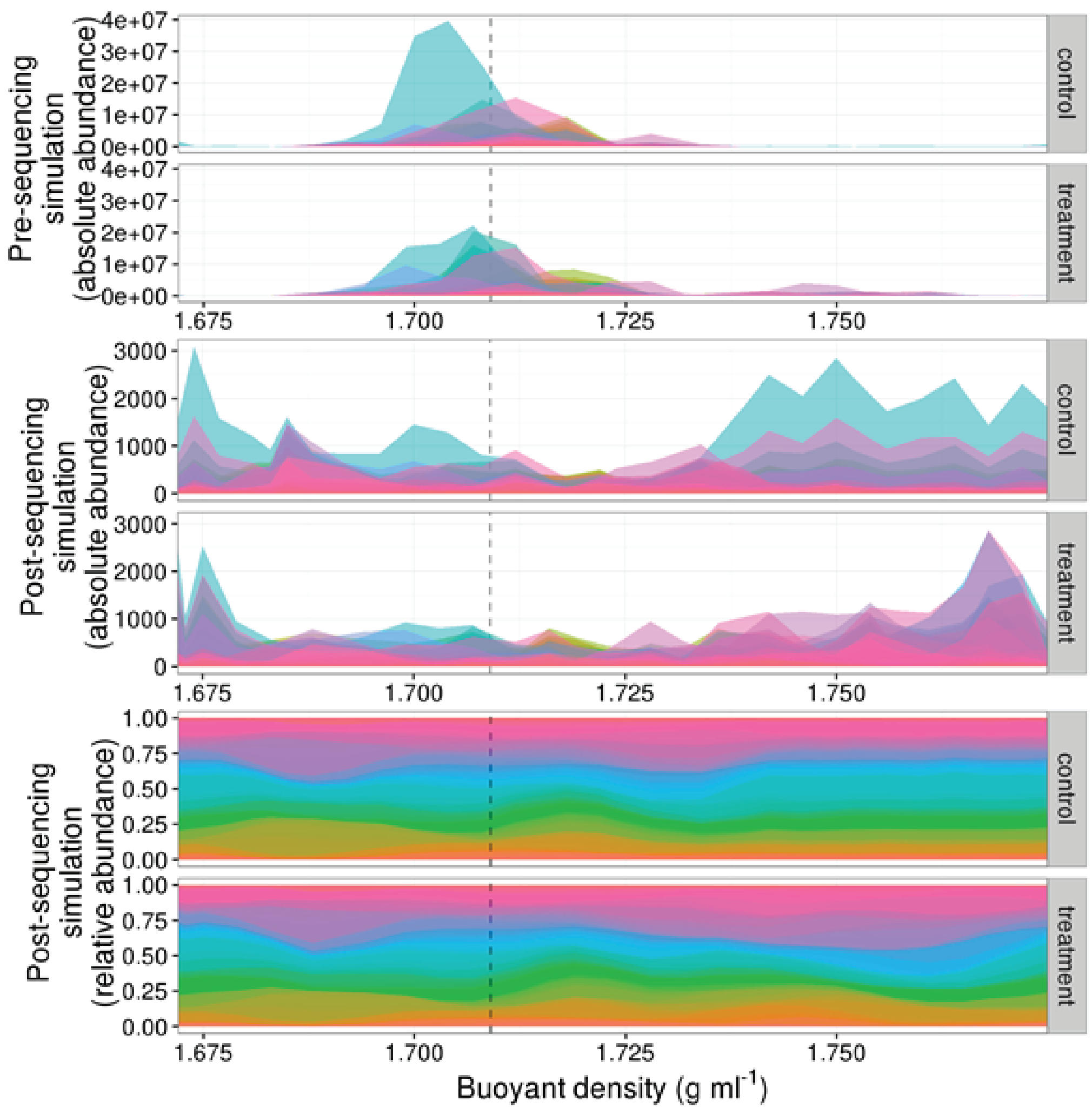




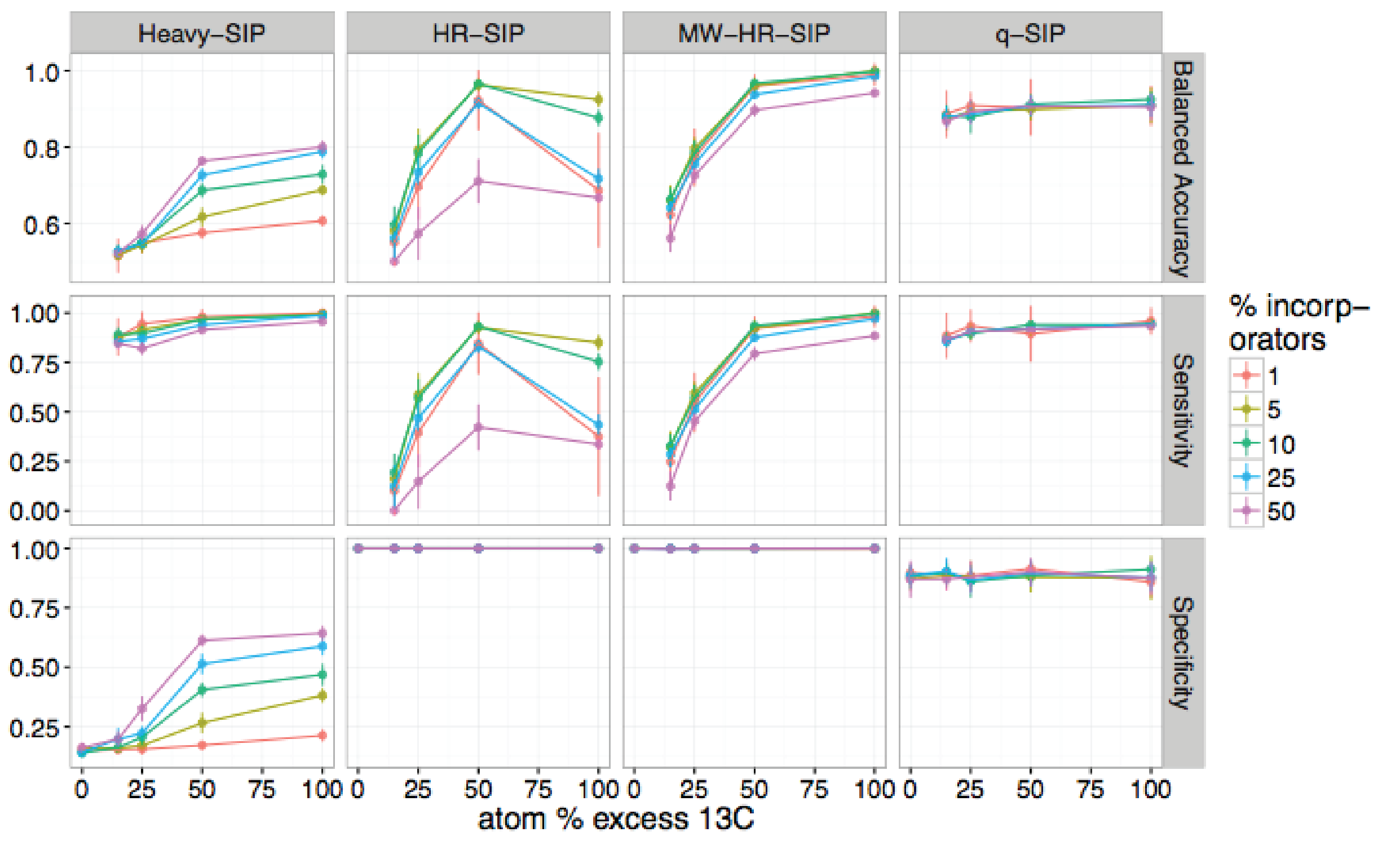




\section{Heavy-SIP}
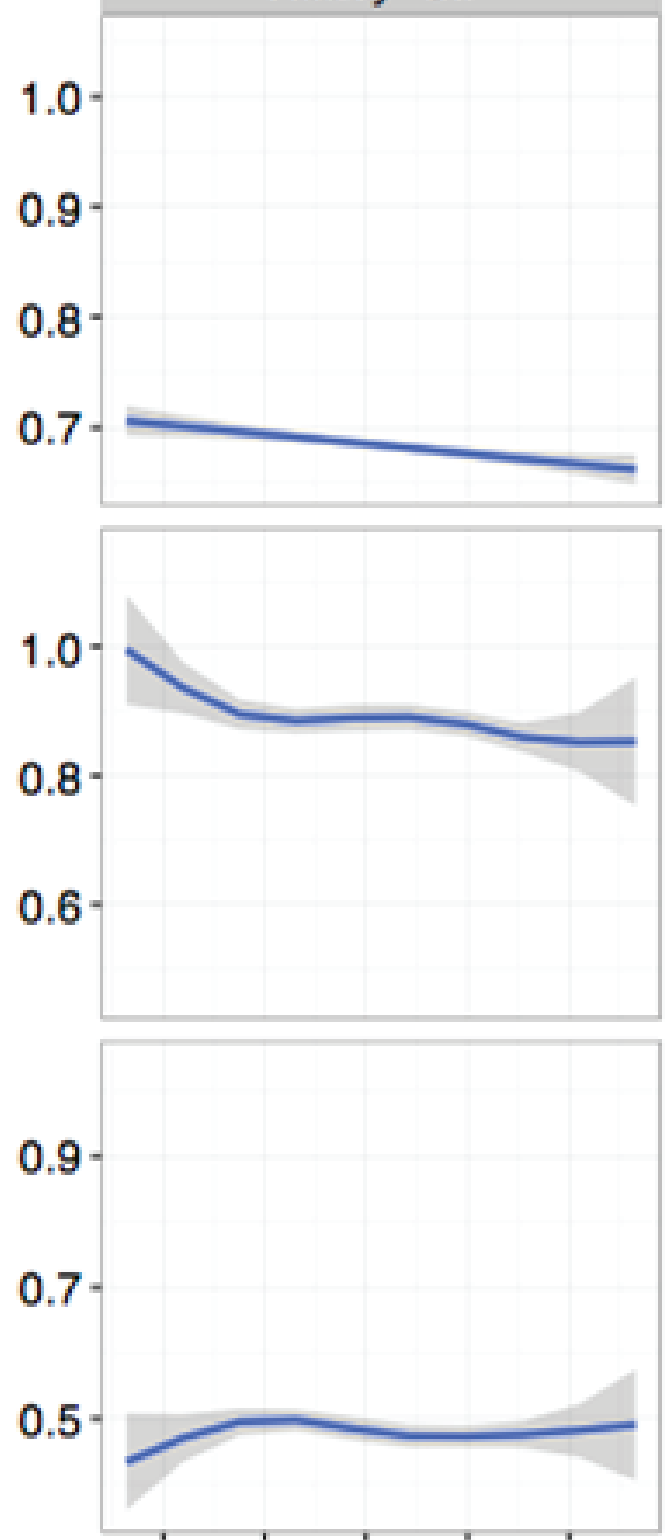

$\begin{array}{lllll}0.1 & 0.2 & 0.3 & 0.4 & 0.5\end{array}$
HR-SIP
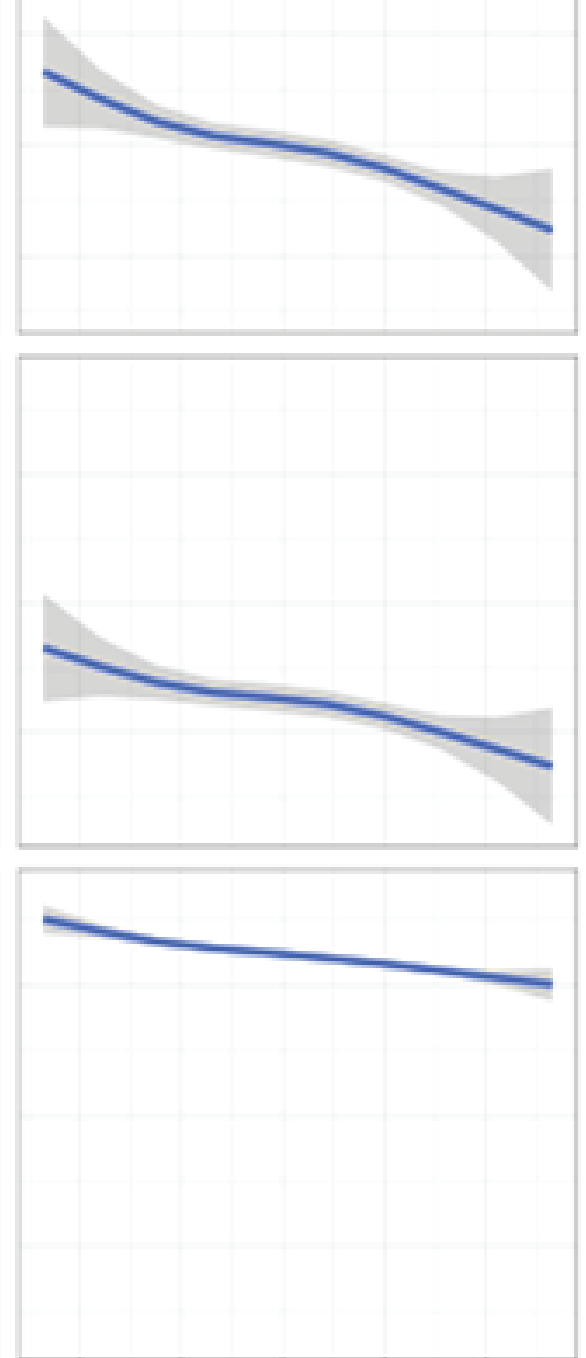

$\begin{array}{lllll}0.1 & 0.2 & 0.3 & 0.4 & 0.5\end{array}$
MW-HR-SIP
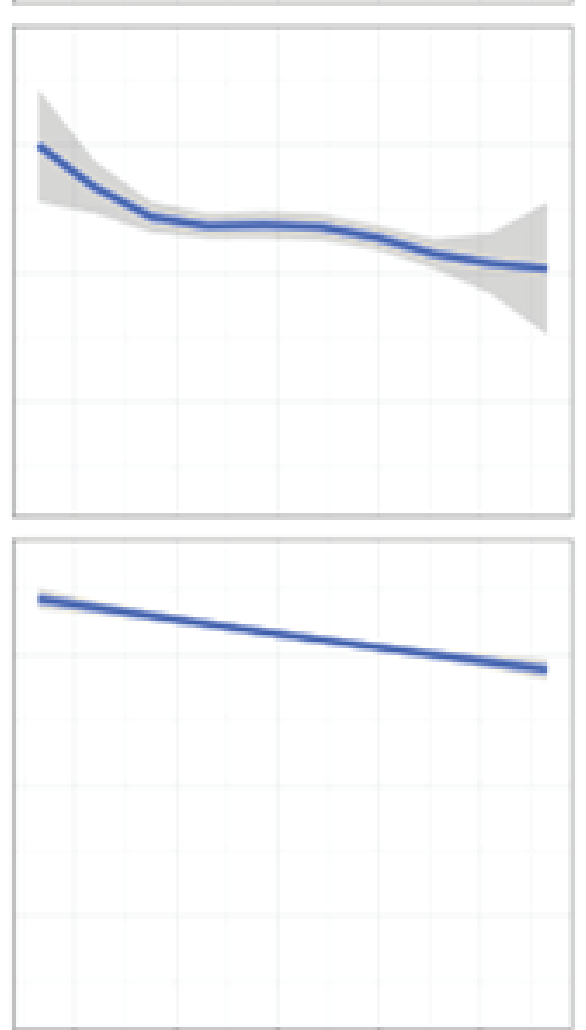

$\begin{array}{lllll}0.1 & 0.2 & 0.3 & 0.4 & 0.5\end{array}$ Mean Bray-Curtis distance among pre-fractionation communities
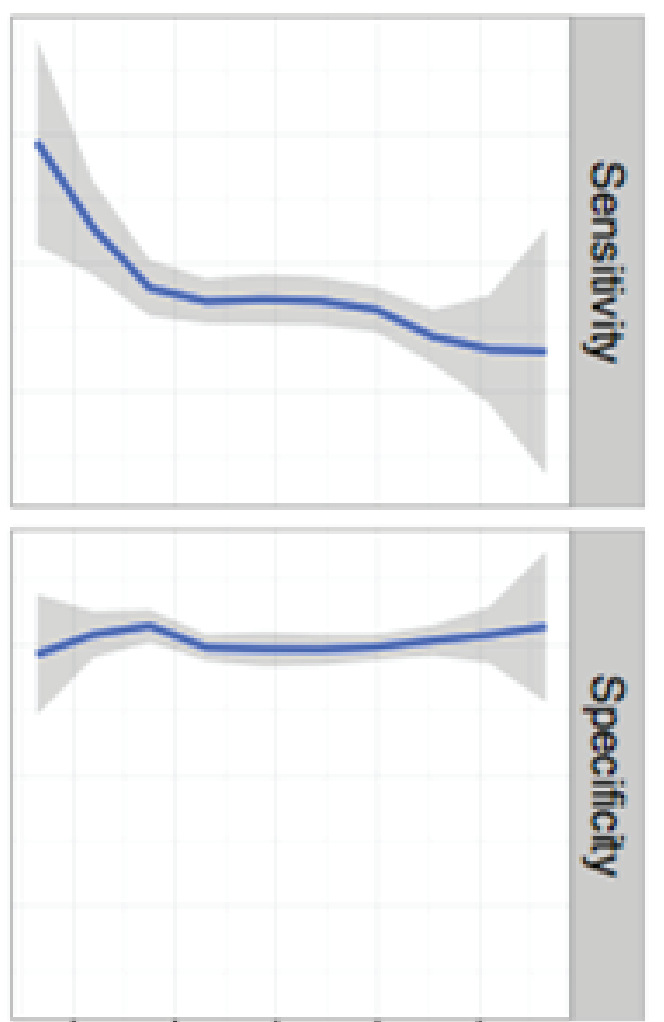

$\begin{array}{lllll}0.1 & 0.2 & 0.3 & 0.4 & 0.5\end{array}$ 


\section{A)}

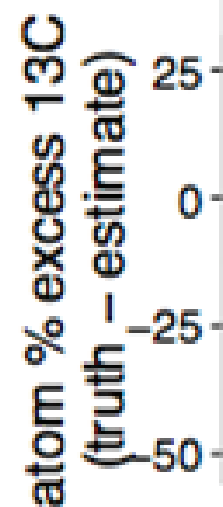

Delta BD

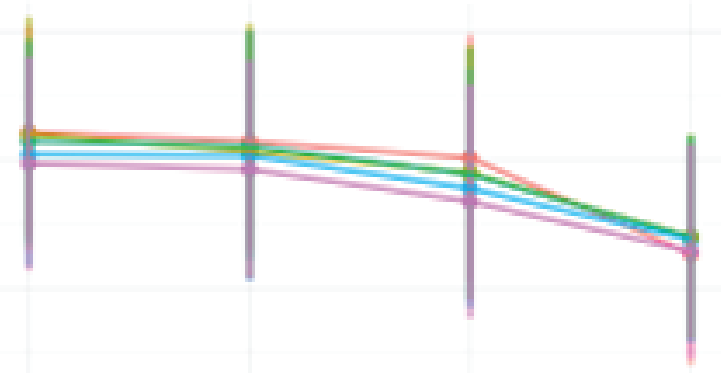

15

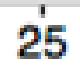

50
q-SIP

B)
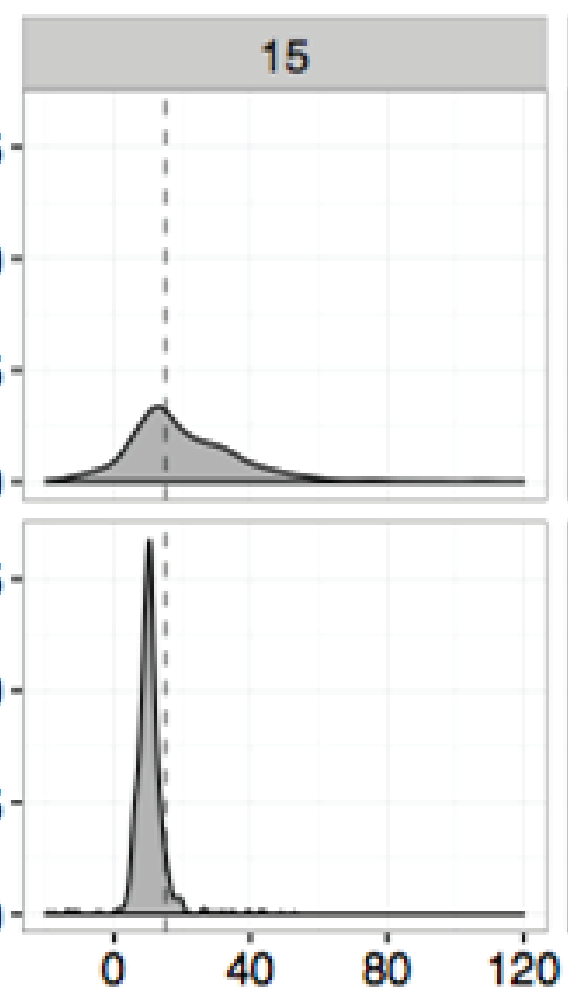
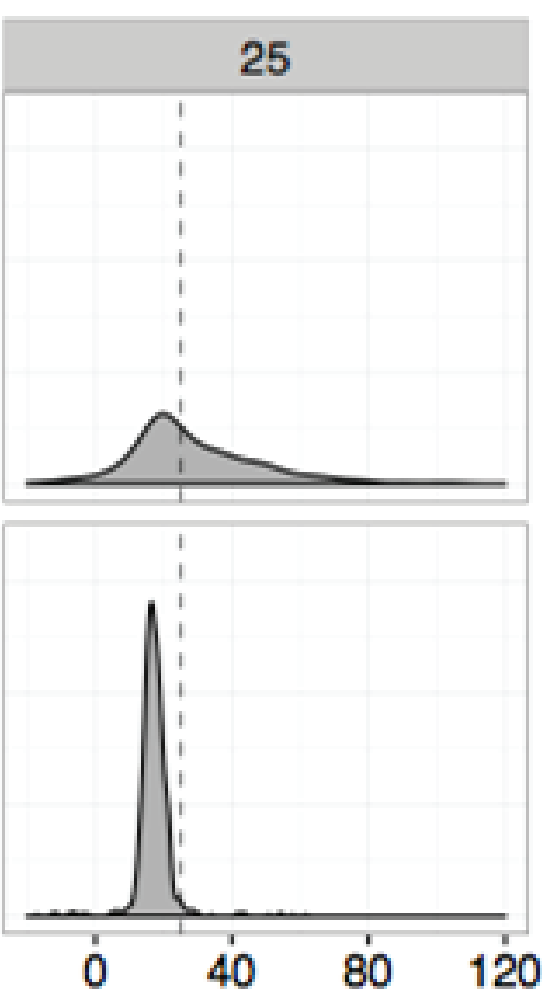
atom $\%$ excess $13 \mathrm{C}$ (estimate)
$\%$ taxa as incorporators

15

525
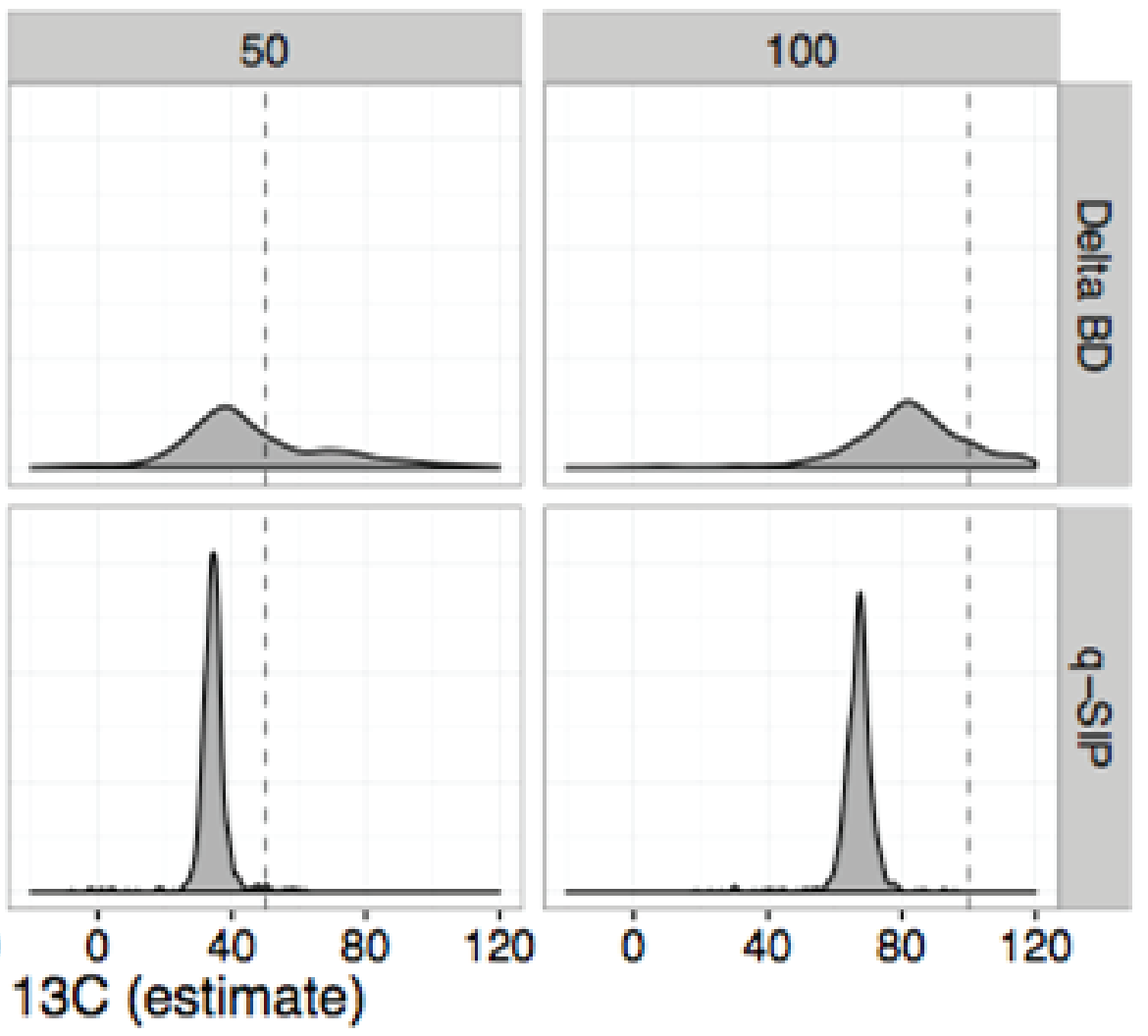